\title{
Comparison of Surveillance of Surgical Site Infections by a National Surveillance Program and by Institutional Audit
}

\author{
Sandra Keller, ${ }^{1, *}$ Fabian Grass, ${ }^{2, *}$ Franziska Tschan, ${ }^{1}$ Valérie Addor, ${ }^{2}$ Christiane Petignat, ${ }^{3}$ \\ Estelle Moulin, ${ }^{3}$ Guido Beldi, ${ }^{4}$ Nicolas Demartines, ${ }^{2}$ and Martin Hübner ${ }^{2}$
}

\begin{abstract}
Background: Reported incidence of surgical site infections (SSI) after colonic surgery varies widely. These variations depend not only on patient- and surgery-related parameters but are influenced by type and quality of follow-up. The aim of the study was to compare SSI assessed by two independent prospective surveillance systems, a national surveillance program based on recommendations of the National Healthcare Safety Network (Swissnoso) versus an international audit system, the ERAS ${ }^{\circledR}$ Interactive Audit System (EIAS; Encare, Stockholm, Sweden). Methods: Comparative study of a consecutive cohort of colonic resections at a single institution from September 2015 to March 2017. Independent prospective SSI monitoring was available from Swissnoso and EIAS. Inter-observer reliability was calculated using Cohen k. Sensitivity, specificity, and accuracy of EIAS in assessing SSI was compared with Swissnoso, considered as gold standard.

Results: The final sample included 143 patients. Of these, $136(95.1 \%)$ were classified into the same category by both systems, identifying 17 patients $(12.5 \%)$ with SSI and 119 patients $(87.5 \%)$ without SSI, respectively. Discrepant results were found for the remaining seven patients (4.9\%) with four SSI categorization according to Swissnoso but not EIAS, and three SSI categorization in EIAS but not in Swissnoso; all miscategorized patients presented superficial SSI. Sensitivity, specificity, and accuracy of EIAS for SSI recording was $81 \%, 97.5 \%$, and $95.1 \%$, respectively. Inter-observer agreement was high (Cohen $\mathrm{k}$ value of $0.801, \mathrm{p}<0.001$ ). Case-by-case analysis of discrepant findings revealed mainly discrepant interpretation of clinical symptoms and erroneous labeling of non-procedure-related infections.

Conclusions: Surgical site infection recording by two independent systems showed high concordance and good inter-rater reliability.
\end{abstract}

Keywords: colonic surgery; enhanced recovery; surgical site infection; surveillance

$\mathbf{S}_{\mathrm{p}}^{\mathrm{s}}$ URGICAL SITE INFECTIONS are the most common complication after colonic surgery and have a major impact on length of stay and costs $[1,2]$. Surgical site infection (SSI) rates are influenced by patient- and surgery-related factors, but they also depend on the quality of the surveillance method [3]. Thus, seemingly good results might also be caused by low-quality surveillance [4]. Accuracy and completeness of assessment during follow-up have important implications for benchmarking, financial decisions, and the perceived need for quality improvement projects [5]. Thus, accurate surveillance of SSI is a first and necessary component of initiatives targeting a reduction of surgical complications [6].
The Enhanced Recovery After Surgery (ERAS) program is a recently initiated international program that provides extensive protocols for pre- and post-operative patient care based on empirical evidence [7]. Surgical site infections are one of the complications targeted by protocols available for elective colonic surgeries [7,8]. Part of ERAS is the ERAS ${ }^{\circledR}$ Interactive Audit System (EIAS; Encare, Stockholm, Sweden), designed to document the compliance with ERAS recommendations, but also to monitor the patient's postsurgical complications, including SSI.

However, the accuracy of EIAS in detecting SSI or other complications has rarely been assessed [9]. The primary aim

\footnotetext{
${ }^{1}$ Institute of Work and Organizational Psychology, University of Neuchâtel, Neuchâtel, Switzerland.

${ }^{2}$ Department of Visceral Surgery, ${ }^{3}$ Department of Hospital Preventive Medicine, Lausanne University Hospital CHUV, Lausanne, Switzerland.

${ }^{4}$ Department of Medicine and Visceral Surgery, Bern University Hospital, University of Bern, Bern, Switzerland.

*Co-first authorship.
} 
of the present study was to compare SSI assessed by EIAS to SSI measured by the national infection surveillance system in Switzerland, Swissnoso (www.swissnoso.ch). Secondary aim was to identify potential sources of erroneous documentation.

\section{Patients and Methods}

\section{Patients and ethical considerations}

Included were all patients undergoing elective and emergent colonic resections between September 1, 2015 and March 31, 2017 at a single institution (Department of Visceral Surgery, Lausanne University Hospital CHUV) that were assessed by both surveillance systems. With 1,500 beds and serving an area of 750,000 inhabitants, CHUV is a medium-sized hospital. All patients included in the study were treated within a standardized enhanced recovery after surgery (ERAS) protocol [8].

This study was part of a larger study that received ethical approval by the Institutional Review Board of the canton of Vaud (CER-VD \#2016-00991) under the leading ethics committee of the canton of Bern, Switzerland (KEK-BE \#161/2014). Patients provided general written informed consent for study participation, including follow-up. In case of missing information on general consent, the leading and local ethics committees approved the use of patient's data.

\section{Demographic, surgery-related, and outcome data}

Demographic information included age, gender, body mass index (BMI), and American Society of Anesthesiologists (ASA) score. Surgical information included approach (open vs. laparoscopic), whether a conversion took place, setting (elective vs. emergency: within 72 hours after unplanned admission), and duration of procedure (time from skin incision to skin closure). Length of hospital stay (LOS) was also assessed.

According to institutional guidelines for colonic resections, intravenous cefuroxime $1.5 \mathrm{~g}$ and metronidazole $500 \mathrm{mg}$ were systematically administered within 60 minutes before incision. As an alternative in case of non-tolerance, clindamycin $600 \mathrm{mg}$ and ciprofloxacin $400 \mathrm{mg}$ were used.

\section{Surveillance systems: Swissnoso and EIAS}

The Swissnoso surveillance system has provided prospective monitoring of SSI by the independent national infection surveillance committee since 1998. This assessment of SSI is based on recommendations of the National Healthcare Safety Network infection tracking system [4]. Surgical site infections were coded according to Swissnoso guidelines by one of two clinical study nurses trained by Swissnoso. They were not associated with the Department of Visceral Surgery and were blind to the hypothesis of this study. Surgical site infections were assessed in-hospital, based on chart reviews, and post-discharge based on phone call at post-operative day 30. Methodological details of this assessment have been published before $[5,10,11]$. Surgical site infections were classified according to the U.S. Centers for Disease Control and Prevention (CDC) National Nosocomial Infection Surveillance (NNIS) criteria into superficial incisional (type 2), deep incisional (type 3), and organ space infections (type 4) [12]. For the purpose of the present study, these categories were grouped into a single category (SSI yes/ no). Diagnostic CDC criteria for superficial incisional infections were further coded as: B1, purulent discharge; B2, positive culture; B3, presence of at least one of the following signs: pain, swelling, redness, warmth, and deliberate wound opening by surgeon; and $\mathrm{C}$, diagnosis by general practitioner [13]. Because the Swissnoso assessment follows the CDC recognized methodology, it was considered the gold standard in this study.

The Interactive Audit System (EIAS), is based on institutional data derived from the ERAS online data repository. Demographic, surgical, and outcome data were monitored prospectively and entered by two clinical study nurses in the EIAS database. For every patient included, a study nurse trained in the EIAS system coded patient data. Systematic assessment was performed during hospitalization and as part of post-operative outpatient visits. Data entry accuracy was cross-checked through regular audit meetings within the department. Infectious complications were classified within EIAS according to the following stratification: wound infection, intra- or retroperitoneal abscess, and anastomotic leak. The study nurse was blind to the results of the Swissnoso assessment and blind to the hypotheses of this study. Table 1 provides a comparative descriptive overview of both surveillance systems.

\section{Outcomes/study end points}

The primary end point was SSI rate (in-hospital and until post-operative day 30) according to surveillance system

Table 1. Comparison of Surveillance Systems

\begin{tabular}{lll}
\hline & \multicolumn{1}{c}{ Swissnoso } & \multicolumn{1}{c}{ EIAS } \\
\hline Assessment scale & National & Institutional \\
Aim & SSI surveillance & Database on peri-operative care \\
Team & Two clinical study nurses & Two clinical study nurses \\
Mode of assessment & Prospective & In-hospital chart review \\
& Chart review & Outpatient consultation report review \\
& Phone call at POD 30 & According to EIAS coding system \\
Definition of SSI & According to U.S. Centers for Disease & \\
& Control and Infection (CDC) & \\
Surveillance committee & and National Nosocomial Infection & Involved (no independent staff) \\
\hline
\end{tabular}

Comparison of different criteria of assessment systems Swissnoso vs. EIAS.

EIAS =ERAS Interactive Audit System; ERAS=enhanced recovery after surgery; POD=post-operative day; SSI=surgical site infection. 


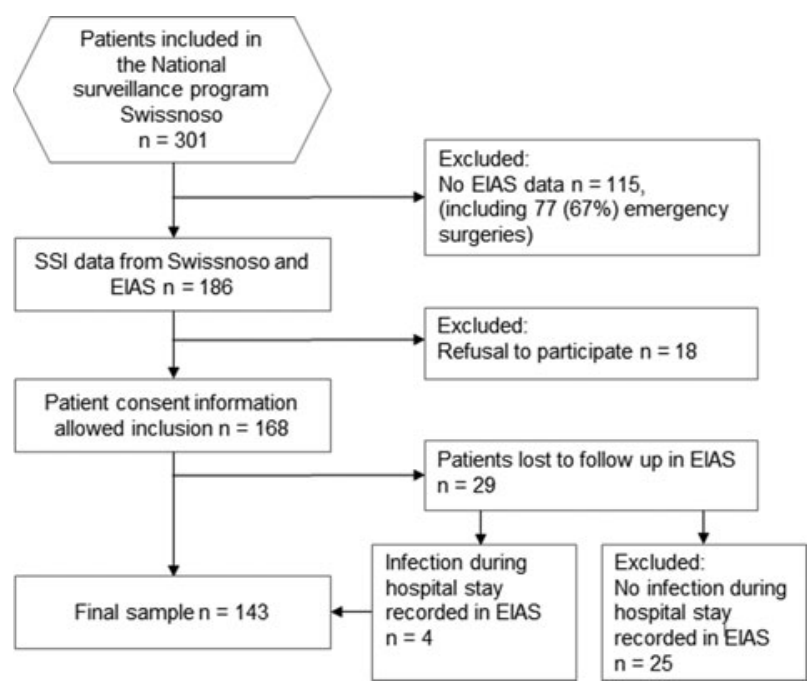

FIG. 1. Patient flow.

(Swissnoso vs. EIAS) and inter-observer reliability between both systems. Discrepancies were evaluated by detailed patient chart review to identify sources of erroneous documentation.

\section{Statistical analysis}

Descriptive statistics for categorical variables were reported as frequency (\%), for continuous variables as means and standard deviations. Discrepancies between the sample and eligible but excluded patients were calculated based on Fisher exact test for categorial variables and Student $t$-test for continuous variables. Inter-observer reliability between the two surveillance systems was assessed using Cohen $\mathrm{k}$ value [14]. The calculation of sensitivity, specificity, and accuracy of EIAS in assessing SSI, compared with Swissnoso, was performed following the standards $[15,16]$. Data analysis was performed with the Statistical Software for the Social Sciences SPSS Advanced Statistics 24 (IBM Corp., Armonk, NY).

\section{Results}

Data from the national surveillance program Swissnoso were available for 301 patients. One hundred fifteen of these patients (38\%) were not part of the ERAS program and were thus excluded; two-thirds of these patients $(\mathrm{n}=77)$ underwent emergency surgery. Therefore, 186 patients $(62 \%)$ were eligible for the present analysis. Of those, 18 patients (10\%) were excluded because of refusal to participate and $29(15 \%)$ were lost to follow-up by the EIAS surveillance system because patients did not attend control visits at the hospital (foreign/out of state). Of these 29 patients, four developed SSI before discharge and were included in the sample. The final sample consisted of 143 patients (Fig. 1).

Demographic and surgical details and LOS are displayed in Table 2 for the patients included in the analysis and as comparison for the 25 patients lost to follow-up by EIAS. Note that none of the characteristics of the excluded patients differed from the sample.

Overall, SSI rate was $15 \%$ according to Swissnoso assessment and $14 \%$ according to EIAS. Of note, among the 25 patients excluded because of insufficient information provided by EIAS, Swissnoso recorded three SSI (12\%).

Cross-tabulation of coded infections according to the two surveillance systems is displayed in Table 3. Inter-observer reliability was high (Cohen $\mathrm{k}$ value of $0.801, \mathrm{p}<0.001$ ). Compared with Swissnoso, EIAS recording had a sensitivity of $81 \%$ (95\% confidence interval $[\mathrm{CI}]=58.09 \%-94.55 \%$ ) and a specificity of $97.5 \%$ (95\% CI $=92.98 \%-99.49 \%)$ for detection of SSI. Positive and negative predictive values were $85 \%(95 \%$ $\mathrm{CI}=64.52 \%-94.64)$ and $96.8 \%(95 \% \mathrm{CI}=92.49 \%-98.63 \%)$, respectively, yielding an accuracy of $95.1 \%(95 \% \mathrm{CI}=90.17 \%$ $98.01 \%)$.

Discrepant findings between both surveillance systems were found in seven patients. Four additional SSI were described according to Swissnoso but not EIAS, and three additional SSI coded in EIAS were not in the Swissnoso database. Details including SSI criteria and potential explanations for erroneous documentation in EIAS are summarized in Table 4. EIAS failed to declare four SSI (patients 1-4), whereas two infections at distant sites were erroneously declared as SSI (patients 5-6). Swissnoso, however, failed

Table 2. Baseline and Surgical Characteristics

\begin{tabular}{lccc}
\hline & Sample $\mathrm{n}=143$ & Patients excluded $^{\mathrm{a}} \mathrm{n}=25$ & $p^{\mathrm{b}}$ \\
\hline Age [y] (mean $\pm \mathrm{SD})$ & $65 \pm 16$ & $62 \pm 19$ & $0.373^{\mathrm{c}}$ \\
Gender male (\%) & $77(54)$ & $14(56)$ & 1 \\
BMI [kg/m ${ }^{2}$ (mean $\left.\pm \mathrm{SD}\right)$ & $26 \pm 6$ & $26 \pm 4$ & $0.985^{\mathrm{c}}$ \\
ASA group (\%) & $95(66)$ & $17(68)$ & 1 \\
I-I & $48(34)$ & $8(32)$ & 1 \\
III-IV & $123(86)$ & $22(88)$ & 0.083 \\
Minimal invasive surgery (\%) & $26(18)$ & $1(4)$ & 1 \\
Conversion (\%) & $28(20)$ & $5(20)$ & $0.909^{\mathrm{c}}$ \\
Emergency surgery (\%) & $170 \pm 80$ & $166 \pm 55$ & $0.98^{\mathrm{c}}$ \\
Operating time [min] (mean $\pm \mathrm{SD})$ & $8 \pm 8$ & $8 \pm 8$ & \\
Length of stay [d] (mean \pm SD) & &
\end{tabular}

Age, BMI, operating time, and length of stay are presented as mean \pm SD. All others are frequency with percentage.

ansufficient information provided by EIAS.

${ }^{\mathrm{b}}$ Fisher exact test, unless indicated otherwise.

${ }^{\mathrm{c}}$ Student t-test.

$\mathrm{SD}=$ standard deviation; $\mathrm{BMI}=$ body mass index; ASA = American Society of Anesthesiologists; EIAS = ERAS Interactive Audit System. 
Table 3. Cross-Tabulation of Coded Infections According to Surveillance Systems

\begin{tabular}{|c|c|c|c|c|}
\hline & \multicolumn{4}{|c|}{ Swissnoso } \\
\hline & & No infection & Infection & Total \\
\hline \multirow[t]{3}{*}{ EIAS } & No infection & 119 & 4 & 123 \\
\hline & Infection & 3 & 17 & 20 \\
\hline & Total & 122 & 21 & 143 \\
\hline
\end{tabular}

EIAS $=$ ERAS Interactive Audit System.

to declare the remaining patient as SSI (patient 7), even though SSI was confirmed through chart review.

Because EIAS follow-up assessment of SSI relied on post-operative consultations rather than the standard assessment 30 days after surgery, we conducted an additional analysis of the days between operation and post-operative consultation. Dates for follow-up were available for 126 patients; for the 13 patients followed by other wards or hospitals, no dates were provided. On average, the post-operative consultations took place 46.06 days after the surgery (standard deviation $[\mathrm{SD}]=13.38$ ).

\section{Discussion}

The present study revealed concordance in SSI assessment between both surveillance systems, Swissnoso and EIAS, yielding good inter-observer reliability. Both systems may thus be considered reliable tools for surveillance of large numbers of surgeries. Whereas EIAS provides more in-depth information on type of infection, fewer patients were lost to follow-up by Swissnoso, because EIAS is solely based on patient information available at the hospital.

Surgical site infection is the most common complication after colorectal surgery with rates of up to $30 \%$ [17] and is a major concern for colorectal surgery [10]. Infection prevention bundles including fostering of a cohesive environment, standardization, and reduction in operative variance have been identified as useful tools to decrease SSI $[18,19]$. However, to assess SSI rates reliably, the quality of follow-up and surveillance is fundamental [3]. Low-quality surveillance might result in falsely low SSI rates, creating drawbacks for patients, caregivers, and policymakers.

Although results yield an overall good inter-rater reliability between the two surveillance systems, four SSIs were missed and three were erroneously coded as SSI in the EIAS database. Of note, all discrepant coding concerned superficial but no deep or organ/space SSI. Detailed chart review of patients with discrepant coding revealed erroneous documentation by ERAS in two cases because EIAS does not include patient follow-up through phone calls. Systematic phone calls as performed by Swissnoso allowed for more complete surveillance. Note, however, that patient reports about SSI have been found to be biased and unreliable $[6,20]$. The other misdiagnosed cases were mainly because of diverging interpretation of clinical symptoms, logistical reasons, or coding mistakes of non-procedure as procedure-

Table 4. Analysis of Divergent Coding

\begin{tabular}{|c|c|c|c|c|c|c|}
\hline \multirow[b]{2}{*}{ Patient } & \multicolumn{4}{|c|}{ Swissnoso } & \multicolumn{2}{|r|}{$E I A S$} \\
\hline & $S S I$ & Type (timing) & Treatment & Criteria/setting & $S S I$ & Explanation \\
\hline 1 & + & Type 2 (POD 13) & No treatment & $\begin{array}{l}\text { B1, B3, C } \\
\text { Phone call post-discharge }\end{array}$ & - & $\begin{array}{l}\text { SSI not detectable applying } \\
\text { EIAS criteria because no } \\
\text { SSI mentioned in surgeons' } \\
\text { follow-up documentation }\end{array}$ \\
\hline 2 & + & Type 2 (POD 10) & Local wound care & $\begin{array}{l}\mathrm{B} 1, \mathrm{~B} 2, \mathrm{~B} 3, \mathrm{C} \\
\text { Inpatient }\end{array}$ & - & $\begin{array}{l}\text { SSI documented in the charts } \\
\text { was interpreted as being } \\
\text { related to another organ } \\
\text { (not related to the current } \\
\text { surgery) }\end{array}$ \\
\hline 3 & + & Type 2 (POD 15) & Surgical management & $\begin{array}{l}\mathrm{B} 1, \mathrm{~B} 2, \mathrm{~B} 3, \mathrm{C} \\
\text { Phone call post-discharge }\end{array}$ & - & $\begin{array}{l}\text { The patient chart correctly } \\
\text { showed SSI. However, the } \\
\text { electronic file was mis- } \\
\text { dated, so that SSI appeared } \\
\text { to have surfaced after } 30 \\
\text { POD }\end{array}$ \\
\hline 4 & + & Type 2 (POD 12) & No treatment & $\begin{array}{l}\text { B1, B2 } \\
\text { Phone call post-discharge }\end{array}$ & - & $\begin{array}{l}\text { Seroma at discharge was } \\
\text { mentioned in the chart but } \\
\text { not diagnosed as SSI }\end{array}$ \\
\hline 5 & - & & & & + & $\begin{array}{l}\text { Non-procedure-related infec- } \\
\text { tion at distant site }\end{array}$ \\
\hline 6 & - & & & & + & $\begin{array}{l}\text { Non-procedure-related infec- } \\
\text { tion at distant site }\end{array}$ \\
\hline 7 & - & & & & + & $\begin{array}{l}\text { Serous fluid excretion through } \\
\text { wound, antibiotic treatment }\end{array}$ \\
\hline
\end{tabular}

Comparison of discrepant cases Swissnoso vs. EIAS regarding SSI documentation.

SSI classification: type 2: superficial incisional.

CDC Criteria: B1: purulent discharge, B2: positive culture, B3: one of the following signs: pain, swelling, redness, warmth AND deliberate wound opening, $\mathrm{C}$ : diagnosis by general practitioner.

EIAS = ERAS Interactive Audit System; SSI = surgical site infection; POD = post-operative day 
related infections, leaving room for improvement in data interpretation and documentation in the EIAS system.

To date, a large variety of surveillance methods has been described [21], but studies assessing comparative reliability of the different tools are still scarce [6,9]. A systematic review across assessment tools revealed problems in compliance with surgeon or patient questionnaires [6], and patients' and surgeons' assessment yielding divergent results. A recent study comparing assessment of complications by EIAS to the National Surgical Quality Improvement Program (NSQIP) methodology stresses the importance of accounting for differences in coding definitions [9). In addition, the different methodologies used complicated the identification of a gold standard [6]. Nevertheless, most reports agree on the need for standardized definitions as basis for a valid approach to survey post-discharge SSI. In the United States, the tracking and reporting of SSI has become an important element for quality improvement purposes. The majority of U.S. hospitals participate in the National Health Safety Network (NHSN) to monitor and report SSI through reporting guidelines [22]. The American College of Surgeons National Surgical Quality Improvement Project (ACS-NSQIP) on the other hand uses trained clinical abstractors and well-defined abstraction guidelines [23]. More recently, a machine learning algorithm has been tested for real-time SSI monitoring, potentially leading to improved surveillance [24].

Both Swissnoso and EIAS are based on standardized definitions of SSI and the assessment process. However, recent analyses revealed a wide variation of SSI surveillance quality in Switzerland, with quality of chart review and accuracy of data collection identified as main areas for improvement [3]. It may be more difficult to establish a high-quality data collection in smaller, rural or non-academic centers, with the risk of less accurate detection of SSI, stressing the importance of standardized training and continuous quality management [25].

Nevertheless, Swissnoso is considered an established SSI surveillance tool due to its National scale and its CDC recognized methodology. Validation of EIAS as SSI surveillance tool is of particular importance since EIAS provides data on perioperative care in general, with SSI being only one among numerous aspects evaluated by the ERAS protocol [9]. The present results are encouraging, considering that both studied surveillance systems were completely independent from each other regarding coding, definitions, assessment methods, and staff.

The study has limitations. First, although data collection was prospective in both surveillance systems, the data analysis for the current study was performed retrospectively, leading to inherent limitations. In particular, the EIAS surveillance system included only patients whose care followed the ERAS protocol guidelines. Patients not formally included in the program, mostly emergencies, were not included in the comparison. Thus, the results cannot be generalized for the surveillance of emergency cases. Second, the cohort was rather small, and data were collected regarding a single type of procedure in one hospital only. Further research is needed to replicate and extend the current findings.

Because both Swissnoso and EIAS rely on detailed protocols to assess SSI and offer standardized training programs for study nurses in charge of the surveillance, bias in the use of the protocol is expected to be minimal. However, mission and methodological approach differ considerably between the two systems, and different definitions of codes may lead to bias [9]. Beyond the accuracy, further criteria may be considered when elaborating recommendations. First, the goal of the surveillance must be considered. Swissnoso offered a comprehensive and systematic surveillance, standardized on the national scale, allowing for comparisons across hospitals. ERAS Interactive Audit System did not offer this possibility, although national and international comparisons may become possible as more hospitals participate in the ERAS program. Second, the assessment relies on resources. Swissnoso surveillance needs specific additional resources in terms of healthcare professionals dedicated to the collection of data not otherwise available, including the patient interview. ERAS Interactive Audit System is based solely on in-hospital and outpatient assessment by treating physicians but not dedicated surveillance specialists and information from patient charts, however, at the cost of a less systematic follow-up and thus a slightly less accurate surveillance.

In conclusion, the present study demonstrated the reliability of EIAS in assessing SSI, providing that patients' follow-up consultations can actually be assessed. Where a nationwide comprehensive surveillance program does not exist, assessment through ERAS data might thus represent a valid alternative.

\section{Acknowledgments}

The authors would like to acknowledge the support provided by the clinical nurses of the Swissnoso surveillance group, Liliana Patricia Moura Pereira, and Homa Salehi Gysei, and all members of the Enhanced Recovery After Surgery team in Lausanne.

This study was supported by a grant from the Swiss National Science Foundation (grant number 156882). The sponsor (Swiss National Science Foundation) had no influence on design of the study, as well as data collection, analysis, or interpretation.

\section{Author Disclosure Statement}

None of the authors declare conflicts of interest.

\section{References}

1. Forbes SS, Stephen WJ, Harper WL, et al. Implementation of evidence-based practices for surgical site infection prophylaxis: Results of a pre- and postintervention study. J Am Coll Surg 2008;207:336-341.

2. Zimlichman E, Henderson D, Tamir O, et al. Health careassociated infections: A meta-analysis of costs and financial impact on the US health care system. JAMA Intern Med 2013;173:2039-2046.

3. Kuster SP, Eisenring MC, Sax H, et al. Structure, process, and outcome quality of surgical site infection surveillance in Switzerland. Infect Control Hosp Epidemiol 2017;38: 1172-1181.

4. Troillet N, Aghayev E, Eisenring MC, et al. First results of the swiss national surgical site infection surveillance program: Who seeks shall find. Infect Control Hosp Epidemiol 2017;38:697-704. 
5. Staszewicz W, Eisenring MC, Bettschart V, et al. Thirteen years of surgical site infection surveillance in Swiss hospitals. J Hosp Infect 2014;88:40-47.

6. Petherick ES, Dalton JE, Moore PJ, Cullum N. Methods for identifying surgical wound infection after discharge from hospital: A systematic review. BMC Infect Dis 2006;6:170.

7. Fearon K, Ljungqvist O, Von Meyenfeldt M, et al. Enhanced recovery after surgery: A consensus review of clinical care for patients undergoing colonic resection. Clin Nutr 2005;24:466-477.

8. Gustafsson UO, Scott MJ, Schwenk W, et al. Guidelines for perioperative care in elective colonic surgery: Enhanced Recovery After Surgery (ERAS ${ }^{\circledR}$ ) Society recommendations. World J Surg 2013;37:259-284.

9. Lyman WB, Passeri M, Cochran A, et al. Discrepancy in postoperative outcomes between auditing databases: A NSQIP comparison. Am Surg 2018;84:1294-1298.

10. Hubner M, Diana M, Zanetti G, et al. Surgical site infections in colon surgery: The patient, the procedure, the hospital, and the surgeon. Arch Surg 2011;146:1240-1245.

11. Romy S, Eisenring MC, Bettschart V, et al. Laparoscope use and surgical site infections in digestive surgery. Ann Surg 2008;247:627-632.

12. Emori TG, Culver DH, Horan TC, et al. National nosocomial infections surveillance system (NNIS): Description of surveillance methods. Am J Infect Control 1991; 19:19-35.

13. Horan TC, Gaynes RP, Martone WJ, et al. CDC definitions of nosocomial surgical site infections, 1992: A modification of CDC definitions of surgical wound infections. Infect Control Hosp Epidemiol 1992;13:606-608.

14. Cohen J. Weighted kappa: Nominal scale agreement provision for scaled disagreement or partial credit. Psychol Bull 1968;70):213-220.

15. Bossuyt PM, Reitsma JB, Bruns DE, et al. STARD 2015: An updated list of essential items for reporting diagnostic accuracy studies. BMJ 2015;351:h5527.

16. Bossuyt PM, Reitsma JB, Bruns DE, Towards complete and accurate reporting of studies of diagnostic accuracy: The STARD initiative. Clin Chem Lab Med 2003;41:68-73.
17. Hewitt DB, Tannouri SS, Burkhart RA, et al. Reducing colorectal surgical site infections: A novel, resident-driven, quality initiative. Am J Surg 2017;213:36-42.

18. Zywot A, Lau CSM, Stephen Fletcher H, Paul S. Bundles prevent surgical site infections after colorectal surgery: Meta-analysis and systematic review. J Gastrointest Surg 2017;21:1915-1930.

19. Cima R, Dankbar E, Lovely J, et al. Colorectal surgery surgical site infection reduction program: A National Surgical Quality Improvement Program -driven multidisciplinary single-institution experience. J Am Coll Surg 2013; 216:23-33.

20. Richter V, Cohen MJ, Benenson S, et al. Patient selfassessment of surgical site infection is inaccurate. World $\mathbf{J}$ Surg 2017;41:1935-1942.

21. Wilson J. Surgical site infection: The principles and practice of surveillance. Part 1: Key concepts in the methodology of SSI surveillance. J Infect Prev 2013;14:6-12.

22. Taylor JS, Marten CA, Potts KA, et al. What is the real rate of surgical site infection? J Oncol Pract 2016;12:e878-e83.

23. Hall BL, Hamilton BH, Richards $\mathrm{K}$, et al. Does surgical quality improve in the American College of Surgeons National Surgical Quality Improvement Program: An evaluation of all participating hospitals. Ann Surg 2009;250:363-376.

24. Sohn S, Larson DW, Habermann EB, et al. Detection of clinically important colorectal surgical site infection using Bayesian network. J Surg Res 2017;209:168-173.

25. Nelson G, Kiyang LN, Crumley ET, et al. Implementation of enhanced recovery after surgery (ERAS) across a provincial healthcare system: The ERAS Alberta colorectal surgery experience. World J Surg 2016;40:1092-1103.

Address correspondence to: Prof. Nicolas Demartines Department of Visceral Surgery Lausanne University Hospital CHUV Lausanne Switzerland

E-mail:demartines@chuv.ch 